\title{
Impacto de Ações Educacionais Sobre o Índice de Higiene Bucal de Escolares de um Município do Sul do Brasil
}

\section{Impact of Educational Actions on the Oral Hygiene Index of Schoolchildren in a Municipality in Southern Brazil}

\author{
Letícia Ribeiro*a; Danielle Gregorio ${ }^{\text {b; }}$ Márjori Frítola Yokoyama ${ }^{c}$; Alessandra Vaz Pinto Hapnerd; \\ Gabriela Fleury Seixas $^{\text {ab }}$; Paulo Christino Neto ${ }^{\mathrm{a}}$; Sandra Mara Maciel ${ }^{\text {ae }}$ \\ ${ }^{a}$ Rede HODOS, Pós-Graduação Lato Sensu em Odontopediatria. PR, Brasil. \\ ${ }^{b}$ Unopar, Programa de Pós-Graduação Stricto Sensu em Odontologia. PR, Brasil. \\ 'Universidade Estadual de Maringá. PR, Brasil. \\ dUniversidade Federal de Santa Catarina. PR, Brasil. \\ eUniversidade Estadual de Maringá, Programa de Pós-Graduação em Odontologia Integrada. \\ *E-mail: leticiaribeiro@ymail.com
}

\begin{abstract}
Resumo
Para evitar doenças como cárie dentária e problemas periodontais é importante estimular hábitos de higiene bucal adequados para que o biofilme seja eficientemente removido pelos indivíduos. O presente estudo objetivou-se a comparar o índice de higiene bucal de um grupo de crianças antes e após estratégias motivacionais de higiene bucal e promoção de saúde. A amostra foi constituída por 43 crianças, 10 a 15 anos, de uma escola pública. Os dentes foram evidenciados com fucsina e o índice de Higiene Oral Simplificado (IHOS) foi avaliado, por um único pesquisador, 7 dias antes (IHOS-1) e 60 dias após (IHOS-2) a uma estratégia de motivação educacional em saúde bucal. Para análise dos dados utilizou-se GraphPad Prism 6 (GraphPad Software Inc., San Diego, CA, EUA), teste de normalidade D’Agostino \& Pearson seguido do teste Wilcoxon para dados não paramétricos, e para paramétricos teste T-Student. Foi possível observar uma redução significativa da presença de biofilme $(p=0,028)$, principalmente no dente $26(p=0,023)$, enfatizando que após a realização de estratégias de promoção de saúde bucal são eficazes. Tanto no IHOS-1 quanto no IHOS-2, a arcada superior foi a arcada com maior presença de biofilme evidenciado $(p<0,001)$. Em relação aos gêneros, não houve diferença significante entre cada avaliação, no entanto, o gênero feminino apresentou redução significante dos valores do índice $(p=0,003)$. Conclui-se que as estratégias educativas- motivacionais, tendo como foco o controle do biofilme dental, impactaram positivamente na prática de higiene bucal pelos escolares.
\end{abstract}

Palavras-chave: Crianças. Saúde Bucal. Higiene Bucal. Placa Dental.

\begin{abstract}
To avoid diseases such as dental caries and periodontal problems, it is important to encourage oral hygiene habits, so that biofilm is efficiently removed by individual. This study aimed to compare the oral hygiene index of a group of children before and after motivational strategies for oral hygiene and health promotion. The sample consisted of 43 children, 10 to 15 years old, from a public school. The teeth were evidenced with fuchsin and the Simplified Oral Hygiene Index (IHOS) was evaluated, by a single researcher, 7 days before (IHOS-1) and 60 days after (IHOS-2) to an educational motivation strategy in oral health. For data analysis, GraphPad Prism 6 (GraphPad Software Inc., San Diego, CA, USA) was used, D'Agostino \& Pearson normality test followed by the Wilcoxon test for non-parametric data, and for parametric T-Student test. It was possible to observe a significant reduction in the presence of biofilm $(p=0.028)$, mainly in tooth $26(p=0.023)$, emphasizing that after carrying out oral health promotion strategies, they are effective. In both IHOS-1 and IHOS-2, the upper arch was the arch with the highest presence of biofilm evidenced ( $p$ 0.001). Regarding genders, there was no significant difference between each evaluation, however, the female gender showed a significant reduction in the index values $(p=0.003)$. It is concluded that educational-motivational strategies, focusing on the control of dental biofilm, had a positive impact on the practice of oral hygiene by students.
\end{abstract}

Keywords: Child. Oral Health. Oral Hygiene. Dental Plaque.

\section{Introdução}

A cárie dentária e a doença periodontal, embora sejam evitáveis, persistem com alta prevalência globalmente, refletindo desigualdades sociais e econômicas e recursos inadequados para prevenção e tratamento, particularmente, em países de baixa e média renda as doenças bucais permanecem, em grande parte, sem tratamento porque os custos do tratamento impõem grandes encargos econômicos às famílias e aos sistemas de saúde e excedem os recursos disponíveis (PERES et al., 2019). Os efeitos negativos destas doenças na infância e adolescência podem se estender à vida adulta e incluem vários aspectos, desde dificuldades funcionais de mastigação, dor, sepse, alterações no sono, redução do apetite e consequente perda de peso, à repercussões no comportamento, como irritabilidade e baixa auto-estima, diminuição de rendimento escolar e de produtividade no trabalho, comprometendo negativamente a qualidade de vida dos indivíduos afetados (PETERSEN; OGAWA, 2016).

Considerada a doença não transmissível mais disseminada, a cárie dentária constitui-se em um dos principais problemas de saúde pública do mundo (WHO, 2017). Segundo o diagrama proposto por Fejerskov e Manji (1990), estão envolvidos no controle e progressão desta doença bucal, além de fatores moduladores como: renda, classe social, atitudes, 
comportamento, conhecimento e educação, os determinantes biológicos, que incluem a saliva, presença de açúcares, microorganismos e de flúor. Em anos recentes, a cárie foi definida como uma desbiose dependente de sacarose e biofilme sobre a superfície dentária, sendo, portanto, imprescindível a presença destes dois fatores na cavidade bucal para o seu desenvolvimento (SHEIHAM; JAMES, 2015). A lesão cariosa só desenvolve e progride em locais onde o biofilme permanece estagnado por um determinado período de tempo, fato resultante da má higiene bucal e baixa exposição ao fluoreto (PITTS et al. 2017).

Vale ressaltar que o biofilme dental é um ecossistema microbiano que permanece aderido à superfície dentária, tendo formação altamente dinâmica (MARSH, 2004; PITTS et al. 2017). E que, também, pode trazer consequências à saúde do periodonto, causando reação inflamatória na região (KINANE; STATHOPOULOU; PAPAPANOU, 2017; MURAKAMI et al., 2018). Dependendo do estado desta inflamação pode causar efeitos sistêmicos, como é o caso da relação entre a doença periodontal e complicações de doenças cardiovasculares (DHADSE; GATTANI; MISHRA, 2010). Controlar a presença do biofilme está diretamente relacionado com hábitos de higiene bucal do indivíduo; apenas uma única escovação por dia é capaz de remover menos de $40 \%$ do biofilme presente na superfície dentária, fato que promove rápido crescimento das bactérias novamente (LA ROSA DE et al., 1979). Evidências científicas atuais preconizam que a higiene bucal adequada para remoção da placa bacteriana deve ser feita pelo menos duas vezes ao dia (ENGLAND, 2017). Programas de educação em higiene bucal são recomendados para causar impacto e motivar as pessoas de acordo com as necessidades individuais (MORAES et al., 2011).

A literatura cita alterações significativas na prevalência e gravidade da cárie dentária relacionadas à adolescência, com aumento significativo entre a idade de 12 anos e a faixa etária de 15 a 19 anos (KASSEBAUM et al., 2017; FIGUEIREDO; BASTOS; PERES, 2017). A pré-adolescência está associada ao aumento do risco e prevalência de doenças bucais como a cárie, por apresentar a associação dos fatores que aumentam a sua susceptibilidade como a independência e aumento do consumo de alimentos açucarados, aliado ao precário controle de biofilme, sendo este consequente à redução dos cuidados com a higienização bucal (SHEIHAM; JAMES, 2015). Além disso, há pouco conhecimento das alterações biopsicossociais, aliado às constantes demonstrações de condutas adaptativas, isto é, mudanças que fazem destes jovens pacientes complexos. (CARVALHO et al., 2011). Dessa forma, é muito importante manter o controle da condição bucal do indivíduo na transição da infância para a adolescência, período em que ocorrem diversas mudanças (TAGLIETTA et al., 2011), mantendo e aprimorando a rotina de higienização bucal. Para atingir este objetivo o desenvolvimento de estratégias educativas em associação ao uso do Índice de Higiene Oral Simplificado
(IHOS) constitui-se em uma abordagem simples e de fácil aplicação (GREENE; VERMILLION, 1960), podendo ser utilizada em escolas de todo o país, podendo ser associada aos programas de promoção de saúde escolar já existentes, trazendo efeito benéfico na conscientização em saúde bucal (WHO, 1999).

$\mathrm{O}$ Índice de Higiene Oral, proposto por Greene e Vermillion, foi primeiramente descrito, em 1960, sendo modificado, posteriormente, pelos mesmos autores em 1964 (GREENE; VERMILLION, 1964), passando a se chamar Índice de Higiene Oral Simplificado (IHOS). O IHOS é amplamente utilizado e indicado para observar a presença de detritos, biofilme, cálculo e até possível relação com doença periodontal (GOMES; SILVA, 2010; FJELD et al., 2018). Além disso, permite uma análise quantitativa simples e sensível para identificar a higiene bucal individual (GREENE; VERMILLION, 1960). Sua aplicação apresenta resultados a curto e longo prazo de programas odontológicos de educação sanitária (GOMES; SILVA, 2010; RÍOS; GARCÍA, 2012; FJELD et al., 2018).

Conhecendo que as atitudes, o conhecimento e a educação do paciente são fatores moduladores para o desenvolvimento de cárie dentária, torna-se importante a orientação e a motivação de impacto, para que cada indivíduo se conscientize da necessidade de realizar a higiene bucal com frequência e acima de tudo, com a indicação da técnica adequada principalmente para crianças, pré-adolescentes e adolescentes. O presente estudo teve o objetivo de avaliar o impacto de estratégias educativas e motivacionais em saúde bucal, associadas ao uso do IHOS, como componente motivacional, no nível de higiene bucal de escolares de um município do sul do Brasil.

\section{Material e Métodos}

O desenvolvimento do estudo atendeu às normas nacionais e internacionais de ética em pesquisa envolvendo seres humanos. O projeto de pesquisa recebeu parecer favorável do Comitê de Ética em Pesquisa local (parecer $n^{\circ} 1.781 .527$ ) e foi autorizado pelo Núcleo Regional de Educação para ser realizado em uma das escolas estaduais da cidade. Os pais/responsáveis pelas crianças foram esclarecidos sobre a pesquisa e assinaram o termo de consentimento livre e esclarecido. O menor também foi informado sobre o estudo sendo solicitado sua assinatura no assentimento livre e esclarecido.

Trata-se de um estudo quase-experimental, do tipo antesdepois com abordagem quantitativa, no qual as aferições foram feitas antes e depois de cada participante ter sido submetido à intervenção. Dessa forma, cada escolar serviu como seu próprio controle para a avalição dos efeitos da intervenção educativa em higiene bucal.

Para o cálculo do tamanho da amostra, assumiu-se um erro $\alpha$ de 0,05 e poder de $90 \%$ em teste post hoc, sendo definida a 
amostra mínima de 42 participantes. Por conveniência, foram inclusos crianças e adolescentes, entre 10 e 15 anos de idade, matriculados no período matutino, no Ensino Fundamental, do Colégio Estadual Dr. Ubaldino do Amaral de Santo Antônio da Platina-PR, no ano de 2016. Foram excluídos os escolares que não se encontravam regularmente matriculados, portadores de necessidades especiais, principalmente com desenvolvimento cognitivo e motor comprometido e aqueles com destruição das faces dos dentes necessários para a determinação do IHOS, assim como os casos de ausência destes em um dos momentos da pesquisa. Os dados foram coletados por um único pesquisador que recebeu treinamento e calibração para realizar a pesquisa.

A calibração do examinador foi realizada com 12 crianças, na idade pré-determinada, nas clínicas Infantil e de Promoção de Saúde da Universidade Tuiuti do Paraná, de modo a mensurar o índice Kappa. O processo de treinamento e calibração ocorreu em duas etapas, sendo a primeira teórica e a segunda prática, realizada em um grupo de crianças com as mesmas características socioeconômicas e etárias da população a ser estudada. A concordância intraexaminadora mínima admitida foi de um valor Kappa de 0,80. O IHOS foi realizado em duas etapas: inicial, previamente aos recursos audiovisuais apresentados às crianças e estratégias motivacionais e final, posteriormente às estratégias. A primeira etapa do estudo incluiu a determinação do IHOS inicial (IHOS - 1) das crianças, para verificar o padrão de acúmulo de placa (biofilme) e a qualidade da escovação. As crianças foram avaliadas em uma sala de aula específica, sob luz natural, individualmente e foi aplicada, sobre todos os dentes, uma solução evidenciadora de biofilme (Fucsina Básica diluída em solução alcoólica 2\% - Eviplac - Biodinâmica - Brasil), com auxílio de hastes flexíveis de algodão.

A evidenciação foi realizada nas seguintes faces dos dentes: Superfícies vestibulares dos dentes 11, 16, 26 e superfícies linguais dos dentes 31, 36 e 46. Com o auxílio de espátulas de madeira esterilizadas, foi realizada a avaliação clínica para verificar a quantidade de placa nestas faces. Determinou-se um escore de 0 a 3 para cada face, sendo 0 para face não corada pelo evidenciador de placa, 1 para as faces coradas na extensão de um terço, escore 2 para as faces coradas na metade da sua extensão e 3 , quando toda a face prédeterminada se apresentou corada. Estes escores foram então somados e divididos por seis, para obter-se o valor do IHOS (GREENE; VERMILLION, 1960)., A partir dos valores do índice procedeu-se a seguinte classificação da higiene bucal dos escolares: boa ( 0,0 a 1,2), razoável (1,3 a 2,0) e deficiente $(2,1$ a 3,0). Foi então determinada à média geral da amostra e esse resultado final foi obtido através das médias aritméticas de placa de todos os participantes da pesquisa, dividida pelo número total da amostra.

Na primeira etapa da pesquisa, foi realizada a evidenciação inicial do biofilme (IHOS-1) que ocorreu após a chegada dos participantes à escola, sem terem realizado nenhuma escovação prévia na escola, antes do momento do lanche escolar e sem que estes tivessem sido avisados sobre o momento e dia do exame clínico. Em seguida ao uso do evidenciador de placa, foram fornecidas escovas dentais (Topz) e dentifrícios fluoretados (Oral B. 1100 ppm) para que estes realizassem a escovação, sem nenhuma orientação ou instrução verbal, para que fosse removido o corante dos dentes.

Sete dias após o IHOS-1, foram aplicadas as estratégias educativo-motivacionais para prevenção da cárie dentária e doença periodontal, pelo controle do biofilme. Procurouse passar aos escolares mensagens simples e claras sobre a higiene bucal adequada para remoção da placa bacteriana (escovar cuidadosamente, com movimentação suave, todas as superfícies dentárias e a margem gengival pelo menos três vezes ao dia, sendo uma delas antes de dormir; usar pouca quantidade de pasta dental, optar por pasta dental fluoretada; escolher escova dental de cabeça pequena e cerdas médias, que deve ser trocada a cada três meses; usar fio dental para limpar os espaços interdentais ao menos uma vez ao dia). Além disso, os alunos também visualizaram as consequências da falha da higienização bucal a partir de imagens, mostrando cavidades de cárie dentária, edema gengival e o sangramento gengival. O conteúdo foi apresentado utilizando recursos audiovisuais, jogos, cartazes e macromodelos, como nos estudos de Aquilante et al. (2003) e Oliveira et al. (2012), para orientação e conscientização de efetuar uma correta higiene bucal com as mesmas crianças que foram avaliadas inicialmente. Um exemplo de jogo, aplicado nesta estratégia, foi uma adaptação do jogo conhecido como "batata quente", uma brincadeira interativa, na qual as crianças passavam um modelo macro de escova, o "escovão", umas para as outras ao som de uma música que era pausada repentinamente. Ao pausar a criança que detinha o objeto era convidada expor os conhecimentos adquiridos no macromodelo, demonstrando aos colegas como seria a maneira correta de higienização. As estratégias foram realizadas em ambiente escolar, nas salas de aula, sendo apresentadas exclusivamente pelo pesquisador, não havendo participação de professores ou cuidadores.

Aproximadamente 60 dias após as estratégias terem sido realizadas, procedeu-se da mesma forma que na análise do IHOS inicial (IHOS-1), para se determinar o IHOS final (IHOS-2). Da mesma forma, os escolares realizaram a escovação após a evidenciação de placa.

Os resultados do IHOS de cada participante, tanto o inicial, quanto o final, foram anotados em uma ficha clínica individual (Figura 1), assim como seus dados pessoais, nome, idade, sexo e dominância manual, ou seja, a mão que a criança utilizava para realizar a escovação (direita ou esquerda). 
Figura 1 - Ficha clínica utilizada para o preenchimento das informações de cada escolar

\section{Ficha Clínica}

Nome:

Idade: $\square 10$ anos $\square 11$ anos $\square 12$ anos $\square 13$ anos $\square 14$ anos

Gênero: $\square$ Feminino $\square$ Masculino

Dominância Manual: $\quad \square$ Destro $\square$ Canhoto

1a Análise do IHOS:

\begin{tabular}{|c|c|c|}
\hline$\square 16 \mathrm{~V}$ & $\square \quad 11 \mathrm{~V}$ & $\square \quad 26 \mathrm{~V}$ \\
\hline$\square 46 \mathrm{~V}$ & $\square \quad 31 \mathrm{~V}$ & $\square \quad 36 \mathrm{~V}$ \\
\hline
\end{tabular}

$2^{a}$ Análise do IHOS:

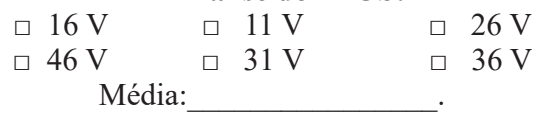

Fonte: Os autores.

Os dados foram processados e analisados pelo programa GraphPad Prism 6 (GraphPad Software Inc., San Diego, CA, EUA, 2012). Inicialmente, procedeu-se ao teste de normalidade D'Agostino \& Pearson, que foi seguido pelo teste Wilcoxon para análise dos dados não paramétricos, e pelo teste T-Student, para dados paramétricos. O nível de significância adotado foi de 5\%.

\section{Resultados e Discussão}

No presente estudo, conduzido em uma escola pública, preencheram os critérios de inclusão, 44 escolares. No entanto, um participante estava ausente no momento da estratégia educativo-motivacional, ficando a amostra final constituída por 43 participantes. Entre estes, a maior proporção tinha 11 anos de idade $(58,1 \%)$ e era do gênero feminino (55,8\%). De acordo com a dominância manual, quase a totalidade (95,3\%) fazia a higienização bucal com a mão direita (Quadro 1).

Quadro 1 - Distribuição dos escolares, segundo a idade, gênero e dominância manual na escovação $(n=43)$. Santo Antônio da Platina - PR, 2016

\begin{tabular}{|l|c|c|}
\cline { 1 - 2 } \multicolumn{1}{|c|}{ Variáveis } & \multirow{2}{*}{ Frequência (N) } & \multirow{2}{*}{ Porcentagem (\%) } \\
\cline { 1 - 2 } 10 anos & 2 & 4,7 \\
\hline 11 anos & 25 & 58,1 \\
\hline 12 anos & 11 & 25,6 \\
\hline 14 anos & 4 & 9,3 \\
\hline 15 anos & 1 & 2,3 \\
\hline \multicolumn{1}{|c|}{ Gênero } & Frequência (N) & Porcentagem (\%) \\
\hline Feminino & 24 & 55,8 \\
\hline Masculino & 19 & 44,2 \\
\hline Dominância Manual & Frequência (N) & Porcentagem (\%) \\
\hline Destro & 41 & 95,3 \\
\hline Canhoto & 2 & 4,7 \\
\hline
\end{tabular}

Fonte: Dados da pesquisa.

A pré-adolescência é uma fase transitória, na qual as crianças já deixam de ser tratadas e cuidadas como tal, para começarem a assumir suas pequenas responsabilidades como adolescentes, entre elas, seus cuidados com sua própria higiene, já que seus pais, não a supervisionam mais, como quando pequenos. Devido a este aspecto, e outros, como autoestima, a fase de transição para a adolescência se constitui em uma fase de mudanças constantes sendo um período propício para motivação e conscientização sobre a prevenção das doenças bucais (TAGLIETTA et al., 2011).

Considera-se relevante para o desenvolvimento de programas de promoção de saúde, o envolvimento de crianças e adolescentes no ambiente escolar, pois esse espaço favorece a disseminação de conhecimentos e a motivação para a adoção de hábitos e atitudes proativas em relação à saúde (PIVOTTO et al., 2013), como hábitos alimentares e de higiene bucal adequados (AQUILANTE et al., 2003; OLIVEIRA et al., 2012; ANTONIO et al., 2015). A escola pública é um alvo de grande relevância, pois possui diferenças socioeconômicas em relação a colégios particulares (COSTA, 2008).

Para avaliação da higiene bucal e controle do biofilme pelo escolar, a média dos IHOS-1 e 2 de cada participante foi calculada, tendo-se observado comportamento normal dos dados, então, para análise estatística utilizou-se o teste T-Student (Tabela 2). Os achados desta análise apontaram para efetividade da intervenção educativo-motivacional conduzida neste estudo, pois foi observada redução significativa da presença de biofilme ( $p=0,028)$, na avaliação final dos escolares. Estudos anteriores, também, demonstraram menores valores do IHOS após o desenvolvimento de ações educativas, que contemplaram conteúdos lúdicos, para a aquisição de corretos hábitos de higiene bucal (SILVEIRA; OLIVEIRA; PADILHA, 2002; AQUILANTE et al., 2003; GITIRANA et al., 2003; GARCIA et al., 2009; MORAES et al., 2011; OLIVEIRA et al., 2012).

Vale destacar que o critério "tempo de investigação após intervenção" deve ser levado em consideração, podendo ter influência nos resultados obtidos. Alguns estudos fizeram a coleta do IHOS após 7, 15 (MORAES et al., 2011), 28 (LA ROSA DE et al., 1979) e 30 dias (GARCIA et al., 2009), ou então, fizeram mais de uma intervenção durante acompanhamento em longos períodos (GITIRANA et al., 2003; OLIVEIRA et al., 2012, OLIVEIRA et al., 2019). Segundo Paredes et al. (2017), nos primeiros dias após a intervenção de orientação de higiene, os cuidados tendem a ser maiores, pelo engajamento imediato. No presente estudo, mesmo decorridos 60 dias das intervenções educativas, bons resultados foram observados, sendo expressos por menor IHOS, ou seja, melhor remoção do biofilme dental, resultante da prática de uma higiene bucal adequada.

Quadro 2 - Média geral e desvio padrão dos valores do IHOS antes e após as estratégias de promoção de saúde, Santo Antônio da Platina, PR, 2016.Platina, PR, 2016

\begin{tabular}{|c|c|c|c|c|}
\hline \multicolumn{2}{|c|}{} & Média & $\begin{array}{c}\text { Desvio } \\
\text { Padrão }\end{array}$ & \multirow{2}{*}{$\boldsymbol{p}^{*}$} \\
\hline \multirow{2}{*}{ IHOS } & IHOS1 & 1,252 & 0,637 & \multirow{2}{*}{$\mathbf{0 , 0 2 8}$} \\
\cline { 2 - 4 } & IHOS2 & 1,065 & 0,587 & \\
\hline
\end{tabular}

$* \mathrm{p}<0,05$. Teste T-Student pareado

Fonte: Dados da pesquisa 
A maioria dos autores faz o uso do IHOS para obter a classificação da higiene bucal (AQUILANTE et al., 2003; GITIRANA et al., 2003; GOMES; SILVA, 2010; MORAES et al., 2011; OLIVEIRA et al., 2012, porém outros utilizam esta mensuração e escores para avaliar a efetividade do controle mecânico do biofilme, desde jovens universitários (RÍOS; GARCIA, 2012) a pré-escolares (CARVALHO et al. 2013). A Figura 2, representa a porcentagem dos escolares deste estudo distribuídos segundo a classificação do IHOS.

Figura 2 - Classificação do nível de higiene dos escolares segundo o IHOS-1 e IHOS -2

๙2

Fonte: Dados da pesquisa.

$\mathrm{Na}$ avaliação inicial, percebe-se proporções similares de escolares classificados como possuindo higiene bucal boa e razoável. Por outro lado, identifica-se que houve um aumento em mais de $10 \%$ do grupo classificado com bom nível de higiene bucal na avaliação final, quando comparada a inicial (Figura 2). No estudo de Ríos e Garcia (2012), o IHOS obtido foi considerado bom para $81,5 \%$ dos participantes. Para Carvalho et al. (2013), somente 8,9\% da amostra apresentaram a higiene considerada boa, a maioria situava-se no escore de IHOS considerado regular $(62,1 \%)$.
$\mathrm{Na}$ comparação entre as arcadas dentárias, quanto a presença do biofilme em cada período avaliado (Quadro 3), foi possível se identificar que a arcada inferior foi significativamente melhor higienizada, quando comparada à superior, tanto na aplicação do IHOS-1 $(p<0,001)$, quanto do IHOS-2 $(p<0,001)$. Estes achados demonstram que durante futuras estratégias motivacionais, provavelmente seja necessário dar mais ênfase e treinamento durante a higienização da arcada superior.

Quadro 3 - Média e desvio padrão da presença do biofilme (IHOS - 1 E 2), segundo as arcadas dentárias

\begin{tabular}{|c|c|c|c|c|}
\hline & \multicolumn{2}{|c|}{ IHOS-1 } & IHOS-2 & \\
\hline Arcada & Média \pm DP & $P$ & Média \pm DP & $P$ \\
\hline Superior & $1,55 \pm 0,86$ & $<$ & $1,372 \pm 0,77$ & $<$ \\
\hline Inferior & $0,96 \pm 0,64$ & 0,001 & $0,79 \pm 0,55$ & 0,001 \\
\hline
\end{tabular}

Teste $\mathrm{t}$ student para dados não pareados. $p<0,05$.

Fonte: Dados da pesquisa.

Registrar o índice de placa apresentado pelo paciente no momento em que ele entra em contato com o profissional tem o intuito de informar a necessidade individual de cada criança e a necessidade de melhorar seu controle de placa e servir como parâmetro de avaliação da melhoria de seu desempenho e numa abordagem coletiva servir como avaliador da eficácia do programa de promoção de saúde (SILVEIRA; OLIVEIRA; PADILHA, 2002).Os valores de mediana obtidos do IHOS1 foram relativamente maiores nos dentes superiores quando comparados aos dentes inferiores. Já, na análise do IHOS-2, observou-se valores de mediana inferiores aos do IHOS-1 na arcada superior, sendo verificada diferença significativa para os o dente 26 ( $p=0,023$ ), da qual, podendo-se inferir que os primeiros molares superiores esquerdos se apresentavam com menor quantidade de biofilme na avaliação final (Quadro 4).

Quadro 4 - Distribuição dos dentes, em relação à quantidade de biofilme, valores mínimos e máximos; mediana e intervalo interquartílicos do escore do IHOS-1 e IHOS-2, nos escolares

\begin{tabular}{|c|c|c|c|c|c|c|c|}
\hline & \multicolumn{3}{|c|}{ IHOS - 1 } & \multicolumn{3}{c|}{ IHOS - 2 } & \multicolumn{1}{c|}{$\boldsymbol{p}$} \\
\hline \multirow{2}{*}{ Dente } & $\begin{array}{c}\text { Mín- } \\
\text { Máx }\end{array}$ & Mediana & $\begin{array}{c}\text { Intervalo } \\
\text { Interquartil }\end{array}$ & $\begin{array}{c}\text { Mín- } \\
\text { Máx }\end{array}$ & Mediana & $\begin{array}{c}\text { Intervalo } \\
\text { Interquartil }\end{array}$ & 0,827 \\
\hline 16 & $0-3$ & 2 & {$[0,0-3,0]$} & $0-3$ & 1 & {$[1,0-3,0]$} & 0,383 \\
\hline 11 & $0-3$ & 2 & {$[0,0-3,0]$} & $0-3$ & 1 & {$[0,0-3,0]$} & $\mathbf{0 , 0 2 3}$ \\
\hline 26 & $0-3$ & 2 & {$[1,0-2,2]$} & $0-3$ & 1 & {$[0,0-2,0]$} & 0,523 \\
\hline 46 & $0-2$ & 1 & {$[0,0-1,0]$} & $0-3$ & 1 & {$[0,0-1,0]$} & 0,297 \\
\hline 31 & $0-3$ & 1 & {$[0,0-3,0]$} & $0-3$ & 1 & {$[0,0-2,0]$} & 0,135 \\
\hline
\end{tabular}

Teste Wilcoxon. $p<0,05$.

Fonte: Dados da pesquisa.

Especula-se diferenças de higienização quanto ao gênero (MARTÍNEZ; HERQUINIO, 2018), porém, alguns estudos não detectaram diferenças estatísticas entre os gêneros dos participantes da amostra (TAGLIETTA et al., 2011; PAREDES et al., 2017; OLIVEIRA et al., 2019; MARTÍNEZ; HERQUINIO, 2018). Na presente pesquisa, considerando-se o gênero do escolar (Quadro 5), na determinação da média geral de IHOS-1, o gênero feminino apresentou maior índice
$(1,35 \pm 0,59)$ em relação ao masculino, sem, contudo, haver diferença estatística ( $p=0,253)$. Da mesma forma, não houve diferença significante entre os gêneros na avaliação do IHOS$2(p=0,788)$. Porém, a comparação entre as avaliações do IHOS-1 e IHOS-2 em cada gênero demonstrou que apenas no gênero feminino houve redução significativa do valor do IHOS ( $p=0,003)$, ou seja, houve melhora nas condições de higiene bucal. 
Quadro 5 - Média e desvio padrão do IHOS 1 e 2 em relação ao gênero

\begin{tabular}{|c|c|c|c|}
\hline & IHOS-1 & IHOS-2 & \\
\hline Gênero & Media \pm DP & Média \pm DP & $\boldsymbol{P}$ \\
\hline Masculino & $1,13 \pm 0,68$ & $1,09 \pm 0,51$ & 0,806 \\
\hline Feminino & $1,35 \pm 0,59$ & $1,04 \pm 0,65$ & $\mathbf{0 , 0 0 3}$ \\
\hline$P$ & 0,253 & 0,788 & \\
\hline *Test & \multicolumn{2}{|l}{} \\
\hline
\end{tabular}

Teste t-Student * $p<0,05$

Fonte: Dados da pesquisa.

Os aparelhos ortodônticos estão associados ao maior acúmulo de biofilme, pela dificuldade de higienização dos acessórios (MEI, L et al. 2017; THENARASU; GURUNATHAN; CHANDRASEKAR,

2018;

VELLIYAGOUNDER et al., 2019), porém mesmo que dois indivíduos do estudo $(4,65 \%)$ tenham colocado aparelho fixo no decorrer das avaliações, os resultados ainda se mostraram positivos, sendo observada redução significante no índice. Por outro lado, nenhuma criança removeu o aparelho, entre as avaliações, o que poderia interferir na avaliação da quantidade de placa entre os dois momentos, podendo apresentar valores menores do índice.

Segundo as observações, a motivação dos pacientes para a construção de hábitos de higiene bucal sempre foi o método mais eficiente na manutenção e na prevenção de doenças bucais, desde que ela ocorra de maneira informal e apropriada para seu público-alvo. Por exemplo, nas escolas, deve-se trabalhar o lúdico para que as crianças tenham interesse em aprender e fazer disto uma rotina (TOASSI; PETRY, 2002; GITIRANA et al., 2003; OLIVEIRA et al., 2012).

As estratégias utilizadas neste estudo foram realizadas com o intuito de motivar os escolares a assumirem os cuidados com sua saúde bucal, criando uma rotina de higienização. Como em estudos prévios (AQUILANTE et al., 2003; OLIVEIRA et al., 2012), as ações educativas foram realizadas com todos escolares reunidos e utilizando-se de palestras e recursos lúdicos como macromodelos, cartazes, fantoches, gincanas e audiovisuais, para efetivar sua motivação. Outras abordagens motivacionais encontram-se relatadas na literatura como o trabalho com gibis (García et al., 2009), show de marionetes (WAMBIER et al., 2013) e vídeo aulas (MARTIGNON et al., 2012).

Deve-se inovar nas práticas educativas, saindo da mesmice da realização de prática de escovação supervisionada e aplicação tópica de flúor, visto que, as motivações devem ser lúdicas e prazerosas, a fim de facilitar a absorção do conhecimento e incentivar a prática de hábito. Muitos são os programas de motivação e educação em relação à higiene bucal com métodos simples e eficientes para a remoção de biofilme dental e prevenção das doenças que este ocasiona. A educação e motivação são capazes de despertar interesse pela manutenção da saúde, desenvolvendo nas pessoas uma consciência crítica das reais causas de seus problemas (OLIVEIRA et al., 2012). No presente estudo, assim como o de Garcia et al. (2009), todos os exames foram realizados sem que os alunos fossem avisados, evitando que os dados obtidos fossem alterados, possibilitando verificar o estado real de higiene bucal dos escolares. Diferentemente, dos estudos de Martignon et al. (2012) que deram instrução verbal antes da escovação, e de Wambier et al. (2013) que a supervisionaram.

A técnica de escovação aplicada nos macromodelos, no momento da motivação para ensinar as crianças, foi a técnica de Bass, pois conforme demonstrou o estudo de Chiarelli, Guimarães e Chaim (2001), esta é 27,67\% mais eficiente na remoção da placa dentária do que a técnica de Fones. Deve-se alertar sobre a sequência de passos feitos durante a higienização, podendo ser até motivo de próximos estudos, visto que, a pessoa que está realizando sua própria higienização bucal pode se cansar e realizar higienização pior em uma determinada arcada ou hemiarco, como demonstrado neste estudo, que a higienização da arcada superior foi significativamente pior do que a da arcada inferior. Devendo, portanto, ser indicada a técnica de higienização que o indivíduo melhor se adapte. Por fim, a prevalência da dominância manual foi avaliada, pois se sugere que os indivíduos que realizam a escovação com a mão direita, o fariam melhor nos dentes do hemiarco esquerdo, como também foi observado neste estudo, maior redução do índice no dente 26). Contudo, não foi possível verificar a relação do nível de higiene com a mão dominante, devido ao pequeno número de participantes canhotos.

A importância de se realizar projetos educativos em saúde bucal é evidenciada neste estudo e também por Oliveira et al. (2019). As estratégias motivacionais também podem ser adaptadas para o consultório odontológico com a intenção de reduzir significativamente a quantidade de biofilme presente na cavidade bucal de pacientes. Considera-se que estas estratégias devam ser repetidas em diversos momentos na vida de um indivíduo para que mantenha seu engajamento e motivação de realizar a correta higienização bucal. Portanto, sugere-se que mais estudos sejam feitos com intervalo ainda maior entre intervenção e coleta de dados, para que seja investigado o engajamento do paciente e motivação de manter sua higiene bucal.

\section{Conclusão}

Conclui-se que as estratégias educativas-motivacionais, tendo como foco o controle do biofilme dental, impactaram positivamente na prática de higiene bucal pelos escolares.

\section{Referências}

ANTONIO, L.P. et al. Avaliação de diferentes métodos educativos em saúde bucal em crianças na faixa etária de 7 a 10 anos de idade. $R F O$, v.20, n.1, p.52-58, 2015. doi: 10.5335/rfo.v20i1.4525

AQUILANTE, A.G. et al. A importância da educação em saúde bucal para pré-escolares. Rev. Odontol. UNESP, v.32, n.1. p.3945,2003

CARVALHO, R.W.F. et al. Aspectos psicossociais dos adolescentes de Aracaju (SE) relacionados à percepção de saúde bucal. Ciênc. Saúde Coletiva, v.16, n.1, p.1621-1628, 2011. doi: 10.1590/S1413-81232011000700098

CARVALHO, T.H.L. et al. Estratégias de promoção de saúde 
para crianças em idade pré-escolar do município de Patos-PB. Rev. Odontol. UNESP, v.42, n. 6, p. 426-431, 2013. doi: 10.1590/ S1807-25772013000600006

CHIARELli, M.; GUIMARÃES, A.; CHAIM, L.A.D.F. avaliação da eficácia das técnicas de escovação dental de bass e fones em relação à remoção de placa, diminuição do sangramento gengival e quantidade de estreptococos mutans e lactobacilos na saliva. Rev. ABO Nac. v.9, n.2, p.88-93, 2001.

COSTA, M. Prestígio e hierarquia escolar: estudo de caso sobre diferenças entre escolas em uma rede municipal. Rev. Bras. Educ., v.13, n.39, p.455-469, 2008. doi: 10.1590/S141324782008000300004.

DHADSE, P.; GATTANI, D.; MISHRA, R. The link between periodontal disease and cardiovascular disease: How far we have come in last two decades? J. Ind. Soc. Periodontol., v.14, n.3, p.148, 2010. doi: 10.4103/0972-124X.75908

ENGLAND, P.H. Delivering better oral health: an evidence-based toolkit for prevention. 2017. Disponível em: www.gov.uk/search ?q=Delivering + better + oral + health $\% 3 \mathrm{~A}+$ an + evidence- based + to olkit+for+prevention+and+Third+edition\&show_organisations_ filter=true

FEJERSKOV, O.; MANJI, F. Risk assessment in dental caries. In: BADER, J.D. Risk assessment in dentistry. 1990. https://www. scienceopen.com/document?vid=8fb76ce8-0641-41b3-90086141 efe 89495

FIGUEIREDO, D. R.; BASTOS, J.; PERES, K. G. Association of adverse oral health outcomes with socioeconomic inequalities and dental needs in Brazilian adolescents. Cad. Saúde Pública, v.33, p. e00165415, 2017. doi: 10.1590/0102-311X00165415

FJELD, K.G. et al. A 1-year follow-up of a randomized clinical trial with focus on manual and electric toothbrushes' effect on dental hygiene in nursing homes. Acta Odontol. Scan., v.76, n.4, p.257-261, 2018. doi: 10.1080/00016357.2017.1416166

GARCIA, P.P.N.S. et al. Educação em saúde: efeito de um método de auto-instrução sobre os níveis de higiene oral em escolares. Pesq. Bras. Odontoped. Clin. Integrad., v.9, n.3, 2009. doi: 10.40 34/1519.0501.2009.0093.0014

GITIRANA, V.F.D. et al. Avaliação de programa de educação odontológico escolar, em crianças de 4 a 5 anos de idade. Rev. Biociênc., v.9, n.4, p.47-51, 2003.

GOMES, V.E.; SILVA D.D. A importância do controle de placa dental na clínica odontológica. Arq. Odontol., v. 46, n. 1, 2010.

GREENE, J.C.; VERMILLION, J.R. The oral hygiene index: a method for classifying oral hygiene status. J. Am. Dental Assoc., v.61, n.2, p.172-179, 1960. doi: 10.14219/jada.archive.1960.0177

GREENE, J.C.; VERMILLION, J.R. The simplified oral hygiene index. J. Amer. Dent. Ass., v.68, p.25-31, 1964. doi: 10.14219/ jada.archive.1964.0034

KASSEBAUM, N. J. et al. Global, regional, and national prevalence, incidence, and disability-adjusted life years for oral conditions for 195 countries, 1990-2015: a systematic analysis for the global burden of diseases, injuries, and risk factors. J. Dental Res., v.96, n.4, p.380-387, 2017. doi: 10.1177/0022034517693566

KINANE, D.F.; STATHOPOULOU P.G.; PAPAPANOU P.N. Periodontal diseases. Nat. Rev. Dis. Primers, v.3, n.1, p.1-14, 2017. doi: $10.1038 /$ nrdp. 2017.38

LA ROSA DE, M. et al. Plaque growth and removal with daily toothbrushing. J. Periodontol., v.50, n.12, p.661-664, 1979. doi: 10.1902/jop.1979.50.12.661

MARSH, P.D. Dental plaque as a microbial biofilm. Caries Res., v.38, n.3, p.204-211, 2004. doi: 10.1159/000077756
MARTIGNON, S. et al. Schoolchildren's toothbrushing characteristics and oral hygiene habits assessed with videorecorded sessions at school and a questionnaire. Acta Odontol. Latinoam., v.25, n.2, p.163-170, 2012.

MARTÍNEZ, D. C.; HERQUINIO, C. C. Asociación entre la higiene oral y la prevalencia de caries dental en escolares de 6 a 12 años de edad de la institución educativa San Gabriel, Villa María del Triunfo, en 2017. Rev. Cient. Odontol., v.6, n.2, p.167176, 2018. doi: 10.21142/2523-2754-0602-2018-167-176

MEI, L. et al. Factors affecting dental biofilm in patients wearing fixed orthodontic appliances. Progress Orthod., v.18, n.1, p.4, 2017.

MORAES, K.R. et al. Motivação de higiene dental utilizando brinquedos com temas odontológicos. Cons. Saúde, v.10, n.4, p.723-728, 2011. doi: 10.5585/conssaude.v10i4.2836

MURAKAMI, S. et al. Dental plaque-induced gingival conditions. J. Periodontol., 2018. doi: 10.1002/JPER.17-0095

OLIVEIRA, M. F. et al. Motivação no controle do biofilme dental e o aprendizado em relação à saúde bucal em escolares. Publ. UEPG Ciênc. Biol. Saúde, v.18, n.2, p.115-120, 2012. doi: 10.5212/publicatio\%20uepg.v18i2.2957

OLIVEIRA, M. F. et al. Avaliação do índice de placa visível antes e depois de sessões de educação em saúde bucal com crianças. Rev. Aten. Saúde, v.17, n.60, p.37-46, 2019. doi: 10.13037/ras. vol17n60.5429

PAREDES, K.A.I. et al. Índice de higiene oral comunitário en escolares de 12 anos. Rev. Evid. Odontol. Clinic. v.3, n.2, p.4650, 2017.

PERES, M.A., et al. Oral diseases: a global public health challenge. Lancet, v.394, n.10194, p.249-260, 2019 . doi: 10.1016/S0140-6736(19)31146-8

PETERSEN, P.E.; OGAWA, H. Prevention of dental caries through the use of fluoride-the WHO approach. Community Dent Health, v.33, n.2, p.66-68, 2016. doi: 10.1922/CDH Petersen03

PITTS, N. et al. Dental caries. Nat. Rev. Dis. Primers, v.3, p.17030, 2017. doi: $10.1038 /$ nrdp. 2017.30

PIVOTTO, A. et al. Hábitos de higiene bucal e índice de higiene oral de escolares do ensino público. Rev. Bras. Promoc. Saúde, v.26, n.4, p.455-461, 2013. doi: 10.5020/18061230.2013.p455

RÍOS, N.I.G; GARCÍA, M.H.M. Determinación de los Índices CPO-D e IHOS en estudiantes de la Universidad Veracruzana. Rev. Chil. Salud Públ., v.16, n.1, p.26-31, 2012.

SHEIHAM, A.; JAMES, W.P.T. Diet and dental caries: the pivotal role of free sugars reemphasized. J. Dental Res., v.94, n.10, p.1341-1347, 2015. doi: 10.1177/0022034515590377

SILVEIRA, J.L.C.G.; OLIVEIRA, V.; PADILHA, W.W.N. Avaliação da redução do índice de placa visível e do índice de sangramento gengival em uma prática de promoção de saúde bucal com crianças. Pesq. Odontol. Bras., v.16, n.2, p.169-174, 2002. doi: 10.1590/S1517-74912002000200013

TAGLIETTA, M. F. A. et al. Impacto de um programa de promoção de saúde escolar sobre a redução da prevalência da cárie em crianças pré-escolares de Piracicaba - SP. RFO, v.16, n.1, p.13-7, 2011.

THENARASU, V.; GURUNATHAN, D.; CHANDRASEKAR, $\mathrm{S}$. Evaluation of plaque index in patient receiving multi-bracket fixed appliance. Res. J. Pharm. Technol., v.11, n.8, p. 3445-3450, 2018. doi: 10.5958/0974-360X.2018.00636.4

TOASSI, R.F.C.; PETRY, P.C. Motivação no controle do biofilme dental e sangramento gengival de escolares. Rev. Saúde Públi., 
v.36, n.5, p.634-637, 2002.

VELLIYAGOUNDER, K. et al. The Microflora Diversity and Profiles in Dental Plaque Biofilms on Brackets and Tooth Surfaces of Orthodontic Patients. J. Indian Orthodontic Soc., v.53, n.3, p.183-188, 2019. doi: 10.1177/0301574219851160

WAMBIER, L. M. et al. The influence of toothbrushing supervision on the dental plaque index and toothbrush wear in preschool children. Rev. Odontol. UNESP, v.42, n.6, p.408-413, 2013. doi: $10.1590 / \mathrm{S} 1807-25772013000600003$
WHO - World Health Organization. Oral health surveys: basic methods. Geneva: WHO, 1997.

WHO - World Health Organization. Improving health through schools: national and international strategies. 1999. Disponível em: https://apps.who.int/iris/bitstream/handle/10665/66314/ WHO_NMH_HPS_00.1.pdf? sequence $=1 \&$ isAllowed $=\mathrm{y}$

WHO - World Health Organization. Sugars and dental caries. 2017. Disponível em: https://apps.who.int/iris/handle/10665/259413 